\title{
Correction to: Features of intracranial hemorrhage in cerebral venous thrombosis
}

\author{
K. Afifi ${ }^{1,25}$ - G. Bellanger ${ }^{2,25} \cdot$ P. J. Buyck ${ }^{3} \cdot$ S. M. Zuurbier ${ }^{4}$. C. Garcia-Esperon ${ }^{5} \cdot$ M. A. Barboza ${ }^{6} \cdot$ P. Costa ${ }^{7}$. \\ I. Escudero ${ }^{8,9} \cdot$ D. Renard ${ }^{10} \cdot$ R. Lemmens ${ }^{11,12,13} \cdot$ N. Hinteregger $^{14} \cdot$ F. Fazekas $^{15} \cdot$ J. Jimenez-Conde $^{16,17}$.

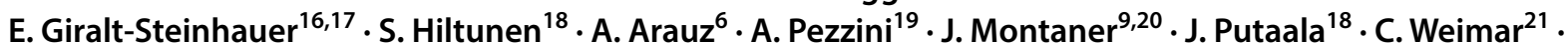 \\ Marc Schlamann $^{22} \cdot$ T. Gattringer ${ }^{15} \cdot$ T. Tatlisumak $^{18,23,24} \cdot$ J. M. Coutinho ${ }^{4} \cdot$ P. Demaerel ${ }^{3} \cdot$ V. Thijs $^{25,26}$
}

Published online: 12 August 2020

(c) Springer-Verlag GmbH Germany, part of Springer Nature 2020

\section{Correction to: Journal of Neurology https://doi.org/10.1007/s00415-020-10008-0}

The original version of this article unfortunately contained mistakes. The correct information is given below.

The original article can be found online at https://doi.org/10.1007/ s00415-020-10008-0.

V. Thijs

vincent.thijs@florey.edu.au

1 Department of Neurology, Menoufia University, Al Minufya, Menoufia, Egypt

2 Department of Neuroradiology, Purpan University Hospital, Toulouse, France

3 Department of Radiology, University Hospitals Leuven, Leuven, Belgium

4 Department of Neurology, Amsterdam University Medical Centers, Location Academic Medical Center, Amsterdam, The Netherlands

5 Department of Neurology, John Hunter Hospital, University of Newcastle, Newcastle, Australia

6 Stroke Department, Instituto Nacional de Neurología Y Neurocirugía Dr. Manuel Velasco Suárez, México City, México

7 Department of Head and Neck, Neurology Clinic, University of Brescia, Brescia, Italy

8 Neurology Department, University Hospital Virgen del Rocio, Sevilla, Spain

9 Neurovascular Lab, Instituto de Biomedicina de Sevilla, Sevilla, Spain

10 Department of Neurology, Nîmes University Hospital, Nîmes, France

11 Department of Neurosciences, Experimental Neurology and Leuven Institute for Neuroscience and Disease (LIND), KU Leuven-University of Leuven, Leuven, Belgium
Family name of following authors C. Garcia-Esperon and J. Jimenez-Conde were incorrect. The correct family name should be Garcia-Esperon and Jimenez-Conde.

12 Laboratory of Neurobiology, Center for Brain and Disease Research, VIB, Leuven, Belgium

13 Department of Neurology, University Hospitals Leuven, Leuven, Belgium

14 Department of Radiology, Division of Neuroradiology, Vascular and Interventional Radiology, Medical University of Graz, Graz, Austria

15 Department of Neurology, Medical University of Graz, Graz, Austria

16 Department of Neurology, Neurovascular Research Group, IMIM-Hospital del Mar, Barcelona, Spain

17 Universitat Autònoma de Barcelon, Barcelona, Spain

18 Department of Neurology, Helsinki University Hospital, Helsinki, Finland

19 Department of Clinical and Experimental Sciences, Neurology Clinic University of Brescia, Brescia, Italy

20 Department of Neurology, Hospital Universitario Virgen Macarena, Seville, Spain

21 Institute of Medical Informatics, Biometry and Epidemiology and Department of Neurology, University Hospital Essen, Essen, Germany

22 Institute for Diagnostic and Interventional Radiology, University of Cologne, Cologne, Germany

23 Department of Clinical Neuroscience/Neurology, Institute of Neuroscience and Physiology, Sahlgrenska Academy at University of Gothenburg, Gothenburg, Sweden

24 Department of Neurology, Sahlgrenska University Hospital, Gothenburg, Sweden

25 Stroke Division, Florey Institute of Neuroscience and Mental Health, University of Melbourne, 245 Burgundy Street, Heidelberg, VIC 3084, Australia

26 Department of Neurology, Austin Health, Heidelberg, VIC, Australia 
The following author's A. Pezzini, J. Montaner, C. Weimar and T. Gattringer affiliations were incorrect. The correct affiliations should be.

\section{A. Pezzini}

Department of Clinical and Experimental Sciences, Neurology Clinic University of Brescia, Brescia, Italy

J. Montaner

Neurovascular Lab, Instituto de Biomedicina de Sevilla, Sevilla, Spain

Department of Neurology, Hospital Universitario Virgen Macarena, Seville, Spain
C. Weimar

Institute of Medical Informatics, Biometry and Epidemiology and Department of Neurology, University Hospital Essen, Essen, Germany

T. Gattringer

Department of Neurology, Medical University of Graz, Graz, Austria 